\title{
Etude Comparative de la Qualite des Eaux des Etangs et du Barrage d'une Ferme Piscicole en Etang au Sud-Est de la Côte d'Ivoire
}

\section{Safiatou Coulibaly, PhD}

Département Aquaculture,

Centre de Recherches Océanologiques, Abidjan, Côte d'Ivoire

Vincent Kouamé N'goran,

Laboratoire d'Hydrobiologie et d'Eco-technologie des Eaux, l'UFR Biosciences, Université Félix HOUPHOUËT-BOIGNY

\section{Célestin Boua Atsé, PhD}

Département Aquaculture,

Centre de Recherches Océanologiques, Abidjan, Côte d'Ivoire

Doi:10.19044/esj.2019.v15n24p42～URL:http://dx.doi.org/10.19044/esj.2019.v15n24p42

\section{Resume}

L'objectif de cette étude est de comparer les paramètres physicochimiques, la concentration des sels nutritifs et de la chlorophylle $a$ des eaux des étangs et du barrage d'alimentation d'une ferme piscicole en étang située à Agboville, au sud-est de la Côte d'Ivoire. Des échantillons d'eau prélevés mensuellement de février à juillet 2017 dans trois étangs de grossissement de Oreochromis niloticus et un barrage d'alimentation ont été analysés selon la norme NF T90-105 de l'AFNOR (1997) pour la détermination de la température, du $\mathrm{pH}$, de la transparence, des matières en suspension, du nitrite, du nitrate, de l'ammonium, de l'orthophosphate et de la chlorophylle $a$. La corrélation entre les variables mesurées est déterminée à l'aide du logiciel Statistica 7.1. Les résultats ont montré que les étangs sont acides, faiblement oxygénés et riches en matière en suspension. Tandis que les eaux du barrage sont pratiquement neutre, oxygénées et transparentes. Le nitrite est pratiquement inexistant aussi bien dans les étangs que dans les eaux du barrage. Les concentrations du nitrate et de la chlorophylle $a$ sont plus élevées dans les étangs qu'au niveau du barrage. Par contre les concentrations de l'ammonium et de l'orthophosphate sont plutôt élevées dans les eaux du barrage. La matrice de corrélation a montré une forte corrélation négative entre la chlorophylle $a$ et l'orthophosphate. En revanche, la chlorophylle $a$ est corrélée positivement avec les matières en suspension et le nitrate. Les résultats montrent que les eaux des étangs sont riches en nutriment pouvant entrainer une prolifération des algues et un début d'eutrophisation. 
Mots clés : Etangs de grossissement, Barrage d'alimentation, Paramètres physico-chimiques, Qualité de l'eau, Ferme piscicole, Côte d'Ivoire

\title{
Comparaive Study of the Water Quality of Ponds and the Dam of a Fish Farm in the Pond in the South-East of Cote d'Ivore
}

\section{Safiatou Coulibaly, PhD}

Département Aquaculture,

Centre de Recherches Océanologiques, Abidjan, Côte d'Ivoire

Vincent Kouamé N'goran,

Laboratoire d'Hydrobiologie et d'Eco-technologie des Eaux, l'UFR Biosciences, Université Félix HOUPHOUËT-BOIGNY

\section{Célestin Boua Atsé, PhD}

Département Aquaculture,

Centre de Recherches Océanologiques, Abidjan, Côte d'Ivoire

\begin{abstract}
The objective of this study was to compare the physicochemical parameters, nutrients and chlorophyll $a$ concentration in waters of ponds and feeding dam of fish farm in Agboville, south-east in Côte d'Ivoire. Water samples collected monthly from February to July 2017 in three Oreochromis niloticus growing ponds and a feed dam were analyzed according to the NF T90-105 standard of AFNOR (1997) for the determination of temperature, $\mathrm{pH}$, transparency, suspended solids, nitrite, nitrate, ammonium, orthophosphate and chlorophyll $a$. The correlation between the variables is determined using the Statistica 7.1 software. The results showed that the ponds are acidic, weakly oxygenated and rich in matter in suspension. While the dam's waters of are practically neutral, oxygenated and transparen. Nitrite is virtually nonexistent in ponds and in the dam. Nitrate and chlorophyll $a$ concentrations are higher in ponds than in the dam. On the other hand, the concentrations of ammonium and orthophosphate are rather high in the dam. The correlation matrix showed a strong negative correlation between chlorophyll $a$ and orthophosphate. In contrast, chlorophyll $a$ is positively correlated with suspended solids and nitrate. The results show that pond waters are rich in nutrients that can lead to algal blooms and eutrophication.
\end{abstract}


Keywords: Growing ponds, Dam Water, Fish farm, Ivory

\section{Introduction}

L'aquaculture est en progression dans de nombreux pays du monde. Elle a pour but de promouvoir la production aquacole afin de répondre à la demande croissante de produits aquatiques (Abboud et al., 2015). Un peu plus de 100 millions de tonnes de poissons sont consommées dans le monde chaque année, et assurent à 2,5 milliards d'êtres humains au moins $20 \%$ de leurs apports moyens par habitant en protéines animales (FAO, 2017). L'aquaculture contribue ainsi à la sécurité alimentaire des populations. En Côte d'Ivoire, elle s'est mise en œuvre grâce aux politiques orientées vers la diversification agricole, l'amélioration des revenus des producteurs et la sécurité alimentaire. Depuis les années 70, ce secteur bénéficie d'une attention particulière dans les politiques nationales de développement économique et social (FAO, 2014). Face à la cherté de la viande, à la moins bonne qualité des poissons importés congelés et aux ralentissements des captures de pêche, les produits de l'aquaculture devraient permettre de répondre à la demande croissante de poisson du marché. Les activités de production animale en milieu aquatique sont donc appelées à jouer un rôle important dans l'économie nationale en termes de création d'emplois, de réduction de la pauvreté, d'amélioration de la productivité, de la compétitivité, de la satisfaction des besoins alimentaires.

Les récents travaux sur la pisciculture ivoirienne révèlent une dominance de l'élevage du tilapia Oreochromis niloticus, majoritairement en étang dans les systèmes semi-intensifs d'élevage (YAO et al., 2016). Dans ces fermes piscicoles, l'alimentation des poissons a été longtemps dominée par l'utilisation massive de sous-produits agroalimentaires contribuant ainsi très peu à la production de poissons marchands compétitifs (Koumi et al., 2015). Ainsi, la production aquacole ivoirienne estimée à 3720 tonnes/an est très faible par rapport à la forte demande qui est d'environ 300000 tonnes/an (FAO, 2014). Cette faible production est en partie liée à la qualité des aliments de poissons (YAO et al., 2016).

Afin d'être plus productives avec de bons rendements, les fermes piscicoles en étang utilisent de plus en plus des aliments commerciaux riches en protéine adaptés à la pisciculture en cage dans les systèmes intensifs. Cependant, ces aliments riches apportent des nutriments (azote et phosphore) qui favorisent la prolifération d'algues dans le milieu d'élevage (Butler et al., 2005) et dégradent la qualité des eaux des fermes piscicoles. Cette situation pourrait porter atteinte à la production aquacole notamment la mortalité des poisons, la baisse de la productivité pouvant aller jusqu'à l'abandon des fermes. 
Cette étude a pour objectif de déterminer la qualité des eaux des étangs de grossissement d'Oreochromis niloticus d'une ferme piscicole utilisant les aliments commerciaux riches en protéines.

\section{I- Materiel et metodes}

\section{I-1-Structures d'élevage des poissons}

Les travaux ont été menés dans les structures de la ferme piscicole ATTA $\left(5^{\circ} 57^{\prime} 14^{\prime \prime} \mathrm{N}\right.$ et $\left.4^{\circ} 27^{\prime} 24^{\prime \prime} \mathrm{W}\right)$ située à Offoumpo à 15 kilomètres d'Agboville (Figure 1). La ferme piscicole compte une quinzaine d'étangs $\left(400\right.$ à $\left.800 \mathrm{~m}^{2}\right)$, trois barrages $\left(8000\right.$ à $12000 \mathrm{~m}^{2}$ ) qui alimentent les étangs et une écloserie constituée de deux bacs en bétons et d'un système de reproduction alimenté par de l'eau de forage. Les étangs sont alimentés en eau par gravité à partir des barrages. Ils sont dotés d'un système de canalisation à ciel ouvert complétés par des tuyaux PVC enterrés qui assurent l'arrivée d'eau dans les structures d'élevage. Ces tuyaux sont équipés d'une toile moustiquaire de protection (maille : $1 \mathrm{~mm}$ ). Un débit d'eau minimal est maintenu dans les étangs, à raison de16 à $20 \mathrm{~m}^{3}$ d'eau /seconde. Les barrages sont alimentés par une rivière en dérivation.
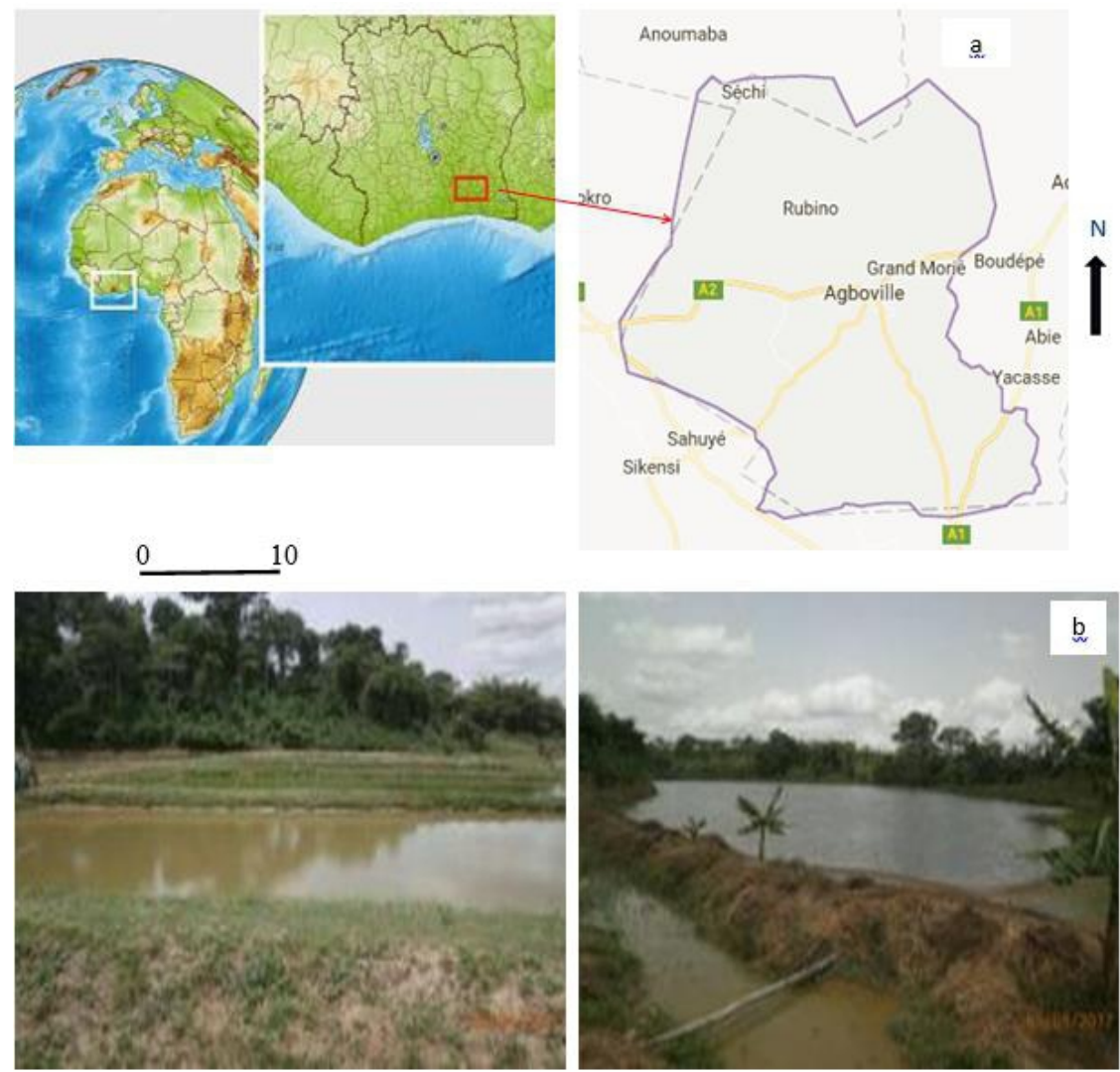

Figure 1: Ferme piscicole ATTA à Offoumpo (Agboville); a: image prise sur Map carte le 16-11-2017 ; b: Photo de Coulibaly Safiatou février 2017 


\section{I-2- Echantillonnage de l'eau et mesure des paramètres physico- chimiques}

Les échantillonnages ont été réalisés mensuellement de février à juillet 2017 dans trois étangs de grossissement et un barrage d'alimentation. Les trois étangs de grossissement ont des superficies variables (étang 1:600 $\mathrm{m}^{2}$, étang $2: 800 \mathrm{~m}^{2}$, étang $3: 700 \mathrm{~m}^{2}$ ) avec une profondeur de $1,50 \mathrm{~m}$ dans la partie la plus profonde. Le barrage a une superficie de $8000 \mathrm{~m}^{2}$ et profond de 2,20 m. Les étangs sont empoissonnés de tilapia Oreochromis niloticus au stade de grossissement (200 à $350 \mathrm{~g}$ ) à la densité de mise en charge de 1 poisson $/ \mathrm{m}^{2}$. Les poissons sont nourris avec de l'aliment granulé flottant extrudé Raanan Fish Feed, importé du Ghana distribuée à la ration de 1\% de la biomasse par jour. Le système d'élevage pratiqué est le système semi intensif.

Le prélèvement des échantillons d'eau a été effectué à l'aide de bouteilles en polyéthylène de un litre de capacité. Les bouteilles sont emballées dans du papier aluminium et transportées dans une glacière à $4{ }^{\circ} \mathrm{C}$ au laboratoire pour les analyses. Au cours des campagnes d'échantillonnage les paramètres physico-chimiques notamment la température, le $\mathrm{pH}$ et l'oxygène dissous ont été mesurés in situ à l'aide d'un multi-paramètre (YSI 6920). La transparence a été déterminée selon la méthode de Secchi. Les mesures ont été effectuées une fois par mois de 8 à 10 heure le matin. Au laboratoire, les prélèvements ont été analysés pour la détermination de la concentration des matières en suspensions selon la méthode décrite par Aminot et Chaussepied (1983). De même, l'analyse de l'orthophosphate $\left(\mathrm{PO}_{4}{ }^{3-}\right)$ a été effectuée à l'aide d'un spectrophotomètre à absorption moléculaire (UV Type 2700) selon Murphy et Riley (1962). Le nitrite $\left(\mathrm{NO}_{2}{ }^{-}\right)$, le nitrate $\left(\mathrm{NO}_{3}{ }^{-}\right)$et l'ammonium $\left(\mathrm{NH}_{4}{ }^{+}\right)$ont été dosés par calorimétrique selon Koroleff (1976). La chlorophylle $a$ a été mesurée après extraction à l'acétone (90\%) par dosage colorimétrique selon Lorenzen (1967).

\section{I-3- Analyse statistique}

L'ANOVA a été utilisée pour évaluer l'effet de la saison sur les paramètres mesurés, et aussi pour évaluer la différence entre le barrage d'alimentation et les étangs de grossissement. Ensuite, le test HSD (Honest Significant Difference) de Tukey a été effectué lorsque l'ANOVA montrait une différence significative. Les différences ont été considérées comme significatives à des valeurs $p<0,05$. La corrélation entre la chlorophylle $a$ et l'orthophosphate a été déterminée. L'analyse statistique a été effectuée à l'aide du logiciel Statistica 7.1. 


\section{II-Resultats et Discussion}

\section{II-1-Résultats}

\section{II-1-1-Variation saisonnière des paramètres physico-chimiques}

Les données des paramètres physico-chimiques sont présentées dans le Tableau I. La température saisonnière est élevée dans toutes les structures. Elle varie de $28,83 \pm 0,13$ à $29,54 \pm 0,13^{\circ} \mathrm{C}$ dans le barrage et de $30,29 \pm 0,19$ à $31,48 \pm 0,20^{\circ} \mathrm{C}$ dans les étangs (Tableau I). Le pH des eaux des étangs $(6,55$ $\pm 0,15-6,99 \pm 0,18)$ a un caractère acide tandis que celui du barrage $(7,12 \pm$ $0,16-7,26 \pm 0,37)$ est faiblement basique. L'oxygène dissous est faible $(3,44$ $\pm 0,16-4,03 \pm 0,66 \mathrm{mg} / \mathrm{L})$ dans les étangs et élevé $(6,12 \pm 0,66-6,39 \pm 0,59$ $\mathrm{mg} / \mathrm{L})$ dans le barrage. La transparence saisonnière est faible dans tous les étangs $(0,50 \pm 0,00-1,50 \pm 0,00 \mathrm{~cm})$. En revanche, dans le barrage, la valeur moyenne de la transparence est plus élevée $(124,00 \pm 0,00-150,00 \pm 0,22 \mathrm{~cm})$. Les concentrations saisonnières des MES sont comprises entre 155,00 $\pm 21,79$ et $268,95 \pm 47,70 \mathrm{mg} / \mathrm{L}$ dans les étangs et entre $93,96 \pm 5,68$ et $97,56 \pm 2,39 \mathrm{mg} / \mathrm{L}$ dans le barrage. L'analyse statistique (ANOVA) montre qu'il n'existe aucune différence significative $(p>0,05)$ entre les saisons pour les paramètres physico-chimiques mesurés à l'exception de la transparence et des MES. Ces deux variables présentent des valeurs basses durant la saison sèche (février, mars et avril) et des valeurs élevées pendant la saison des pluies (mai, juin et juillet) aussi bien dans les étangs que dans le barrage (Tableau I). Par contre, des différences significatives $(p<0,05)$ ont été enregistrées entre les valeurs moyennes de tous les paramètres physico-chimiques mesurés à l'exception du $\mathrm{pH}$ dans les étangs et dans le barrage indépendamment des saisons. Le barrage enregistre des valeurs minimales pour la température et les MES et des valeurs maximales pour l'O ${ }_{2}$ et la transparence.

Tableau 1 : Variations saisonnières et valeurs moyennes de la Température $\left({ }^{\circ} \mathrm{C}\right), \mathrm{du} \mathrm{pH}$, de l'Oxygène dissous $(\mathrm{mg} / \mathrm{L})$, de la transparence $(\mathrm{cm})$ et des Matières en Suspensions $(\mathrm{mg} / \mathrm{L})$ des eaux des structures de la ferme piscicole ATTA de février à juillet 2017.

\begin{tabular}{|c|c|c|c|c|c|}
\hline \multicolumn{6}{|c|}{ Structures } \\
\hline Paramètres & & Barrage & Etang 1 & Etang 2 & Etang 3 \\
\hline \multirow[t]{3}{*}{$\begin{array}{c}\text { Température } \\
\left({ }^{\circ} \mathrm{C}\right)\end{array}$} & SS & $\begin{array}{c}29,54 \pm \\
0,13\end{array}$ & $31,33 \pm 0,31$ & $31,24 \pm 0,17$ & $31,48 \pm 0,20$ \\
\hline & SP & $\begin{array}{c}28,83 \pm \\
0,13\end{array}$ & $30,44 \pm 0,24$ & $30,82 \pm 0,65$ & $30,29 \pm 0,19$ \\
\hline & VM & $\begin{array}{c}29,19 \pm \\
0,40^{2}\end{array}$ & $30,88 \pm 0,55^{1}$ & $31,03 \pm 0,48^{1}$ & $30,88 \pm 0,67^{1}$ \\
\hline \multirow[t]{3}{*}{$\mathrm{pH}$} & SS & $7,12 \pm 0,16$ & $6,99 \pm 0,18$ & $6,55 \pm 0,15$ & $6,60 \pm 0,28$ \\
\hline & SP & $7,26 \pm 0,37$ & $6,78 \pm 0,22$ & $6,62 \pm 0,54$ & $6,72 \pm 0,51$ \\
\hline & VM & $7,19 \pm 0,26$ & $6,89 \pm 0,21$ & $6,58 \pm 0,35$ & $6,66 \pm 0,37$ \\
\hline \multirow[t]{2}{*}{$\mathrm{O}_{2}(\mathrm{mg} / \mathrm{L})$} & SS & $6,12 \pm 0,66$ & $3,61 \pm 0,30$ & $3,44 \pm 0,16$ & $3,62 \pm 0,59$ \\
\hline & SP & $6,39 \pm 0,59$ & $3,88 \pm 0,23$ & $4,03 \pm 0,66$ & $4,03 \pm 0,60$ \\
\hline
\end{tabular}




\begin{tabular}{|c|c|c|c|c|c|}
\hline & VM & $\begin{array}{c}6,25 \pm \\
0,58^{1}\end{array}$ & $3,74 \pm 0,28^{2}$ & $3,73 \pm 0,54^{2}$ & $3,83 \pm 0,60^{2}$ \\
\hline \multirow[t]{3}{*}{$\begin{array}{l}\text { Transparence } \\
\text { (cm) }\end{array}$} & SS & $\begin{array}{c}124,00 \pm \\
0,00^{\mathrm{a}}\end{array}$ & $0,50 \pm 0,00^{\mathrm{a}}$ & $0,50 \pm 0,00^{\mathrm{a}}$ & $0,50 \pm 0,00^{\mathrm{a}}$ \\
\hline & SP & $\begin{array}{c}150,00 \pm \\
0,22^{\mathrm{b}}\end{array}$ & $1,50 \pm 0,00^{\mathrm{b}}$ & $1,10 \pm 0,00^{b}$ & $1,10 \pm 0,00^{b}$ \\
\hline & VM & $\begin{array}{c}137,00 \pm \\
0,20^{1}\end{array}$ & $0,01 \pm 0,00^{2}$ & $0,00 \pm 0,00^{2}$ & $0,00 \pm 0,00^{2}$ \\
\hline \multirow[t]{3}{*}{ MES (mg/L) } & SS & $\begin{array}{c}93,96 \pm \\
5,68^{\mathrm{a}}\end{array}$ & $219,29 \pm 27,27^{\mathrm{a}}$ & $163,09 \pm 21,11^{\mathrm{a}}$ & $155,00 \pm 21,79^{a}$ \\
\hline & SP & $\begin{array}{c}97,56 \pm \\
2,39^{\mathrm{b}}\end{array}$ & $268,95 \pm 47,70^{\mathrm{b}}$ & $216,66 \pm 36,29^{b}$ & $261,33 \pm 11,84^{\mathrm{b}}$ \\
\hline & VM & $\begin{array}{c}95,76 \pm \\
4,36^{2}\end{array}$ & $244,12 \pm 44,13^{1}$ & $189,88 \pm 39,57^{1}$ & $208,16 \pm 60,31^{1}$ \\
\hline
\end{tabular}

$\mathrm{SS}=$ Saison sèche $; \mathrm{SP}=$ Saison des pluies, $\mathrm{VM}=$ valeurs moyennes

Les valeurs de la même colonne portant des lettres $a, b$, en exposant présentent une différence entre les saisons $(p<0,05)$ pour une structure donnée.

Les valeurs de la même ligne portant des lettres 1,2 en exposant présentent une différence des valeurs moyennes entre les structures $(p<0,05)$.

\section{II-1-2-Variations mensuelles et saisonnières des sels nutritifs et de la chlorophylle $a$}

Les données des sels nutritifs et de la chlorophylle $a$ sont présentées dans le Tableau II. Les variations mensuelles des concentrations du nitrite, nitrate, ammonium, orthophosphate et de la chlorophylle $a$ sont comprises respectivement, entre 0,00 et $0,10 \mathrm{mg} / \mathrm{L} ; 3,24$ et $11,21 \mathrm{mg} / \mathrm{L} ; 0,19$ et 4,82 $\mathrm{mg} / \mathrm{L} ; 0,49$ et $5,14 \mathrm{mg} / \mathrm{L} ; 2,03$ et $9,89 \mathrm{mg} / \mathrm{L}$. Au niveau du barrage, les valeurs enregistrées ne présentent aucune différence significative $(p>0,05)$ entre les saisons pour tous les sels nutritifs et pour la chlorophylle $a$. Par contre au niveau des étangs, des différences significatives $(p<0,05)$ ont été enregistrées entre les saisons pour le nitrate, l'orthophosphate et la chlorophylle $a$. Au cours de la saison des pluies, les valeurs du nitrite $(0,01 \pm 0,00 \mathrm{mg} / \mathrm{L})$ et de la chlorophylle $a(8,83 \pm 1,51 \mu \mathrm{g} / \mathrm{L})$ sont plus élevées que celles en saison sèche. En revanche, les valeurs de l'orthophosphate $(0,96 \pm 0,78 \mathrm{mg} / \mathrm{L})$ sont plus faibles en saison de pluies qu'en saison sèche. Les valeurs moyennes montrent une différence significative $(p<0,05)$ entre le barrage et les étangs pour tous les paramètres sauf le nitrite. Les valeurs de nitrate $(9,58 \pm 1,16 \mathrm{mg} / \mathrm{L})$ et de chlorophylle $a(7,24 \pm 2,16 \mathrm{mg} / \mathrm{L})$ sont élevées dans les étangs. En revanche, les valeurs de l'ammonium $(2,96 \pm 0,93 \mathrm{mg} / \mathrm{L})$ et de l'orthophosphate $(2,67 \pm 0,59 \mathrm{mg} / \mathrm{L})$ sont plus élevées dans le barrage. 


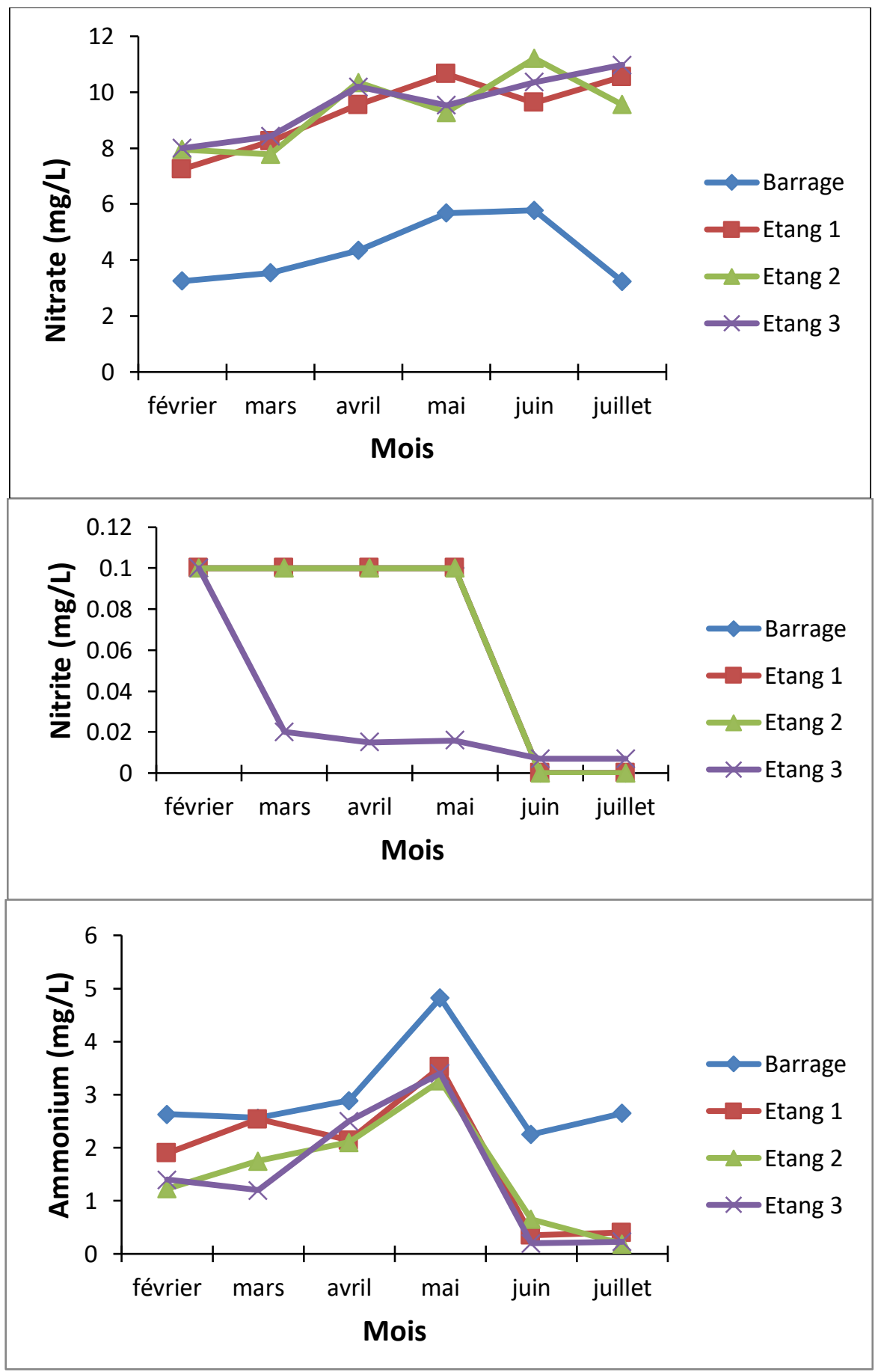

Figure 2: Variation mensuelle du nitrate, nitrite et de l'ammonium en $\mathrm{mg} / \mathrm{L}$ des étangs et $d u$ barrage de la ferme piscicole ATTA de février à juillet 2017. 


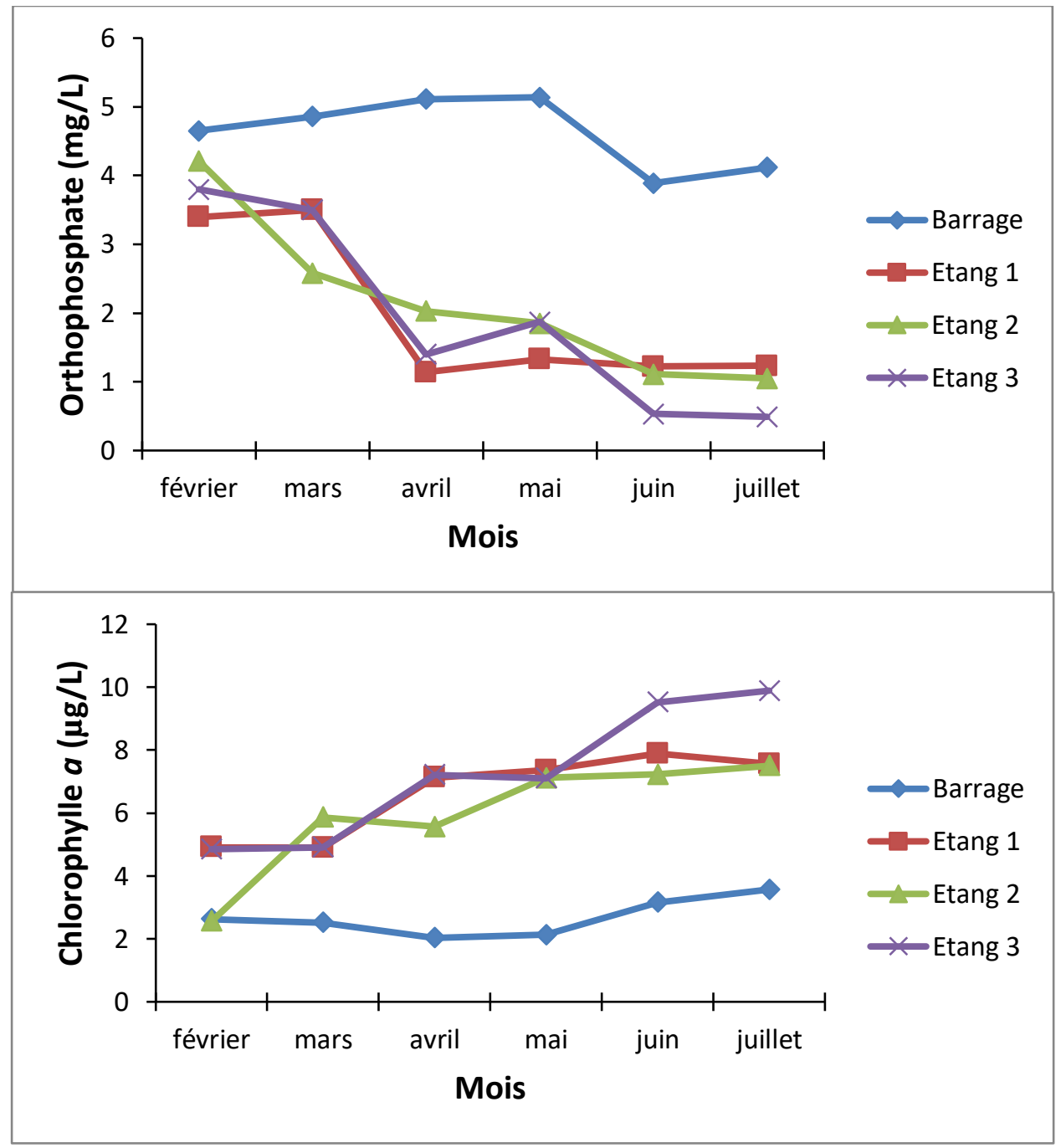

Figure3:Variation mensuelle de l'orthophosphateen $(\mathrm{mg} / \mathrm{L})$ et de la chlorophylle a en $(\mu \mathrm{g} / L)$ des eaux des étangs et du barrage de la ferme piscicole ATTA de février à juillet 2017.

Tableau 2 : Variation saisonnières et valeurs moyennes des sels nutritifs $\left(\mathrm{NO}_{2}{ }^{-}, \mathrm{NO}_{3}{ }^{-}, \mathrm{NH}_{4}{ }^{+}\right.$ et $\left.\mathrm{PO}_{4}{ }^{3-}\right)$ et la chlorophylle a en $(\mu \mathrm{g} / \mathrm{L})$ des eaux des étangs et du barrage de la ferme piscicole ATTA de février à juillet 2017.

\begin{tabular}{|c|c|c|c|c|c|}
\hline \multicolumn{6}{|c|}{ Structures } \\
\hline Paramètres & & Barrage & Etang 1 & Etang 2 & Etang 3 \\
\hline $\mathrm{NO}_{2}^{-}$ & SS & $0,01 \pm 0,00$ & $0,01 \pm 0,00$ & $0,01 \pm 0,00$ & $0,01 \pm 0,00$ \\
\hline & SP & $0,00 \pm 0,00$ & $0,00 \pm 0,00$ & $0,00 \pm 0,00$ & $0,01 \pm 0,00$ \\
\hline & $\mathrm{CM}$ & $0,00 \pm 0,00$ & $0,00 \pm 0,00$ & $0,00 \pm 0,00$ & $0,01 \pm 0,00$ \\
\hline $\mathrm{NO}_{3}^{-}$ & SS & $3,71 \pm 0,57$ & $8,34 \pm 1,15^{a}$ & $\begin{array}{c}8,69 \pm \\
1,43^{\mathrm{a}}\end{array}$ & $\begin{array}{c}8,87 \pm \\
1,16^{\mathrm{a}}\end{array}$ \\
\hline
\end{tabular}




\begin{tabular}{|c|c|c|c|c|c|}
\hline & SP & $4,90 \pm 1,43$ & $\begin{array}{c}10,27 \pm \\
0,56^{\mathrm{b}}\end{array}$ & $\begin{array}{c}10,01 \pm \\
1,04^{\mathrm{b}}\end{array}$ & $\begin{array}{c}10,29 \pm \\
0,72^{\mathrm{b}}\end{array}$ \\
\hline & $\mathrm{CM}$ & $4,30 \pm 1,17^{2}$ & $9,31 \pm 1,33^{1}$ & $\begin{array}{c}9,35 \pm \\
1,33^{1}\end{array}$ & $\begin{array}{c}9,58 \pm \\
1,16^{1}\end{array}$ \\
\hline \multirow[t]{3}{*}{$\mathrm{NH}_{4}^{+}$} & SS & $2,69 \pm 0,17$ & $2,52 \pm 0,88$ & $1,69 \pm 0,43$ & $1,70 \pm 0,70$ \\
\hline & SP & $3,24 \pm 1,38$ & $1,42 \pm 1,81$ & $1,36 \pm 1,65$ & $\begin{array}{c}1,27 \pm 1 \\
, 83\end{array}$ \\
\hline & $\mathrm{CM}$ & $2,96 \pm 0,93^{1}$ & $1,97 \pm 1,41^{2}$ & $\begin{array}{c}1,53 \pm \\
1,09^{2}\end{array}$ & $\begin{array}{c}1,48 \pm \\
1,26^{2}\end{array}$ \\
\hline \multirow[t]{3}{*}{$\mathrm{PO}_{4}^{3-}$} & SS & $4,87 \pm 0,23$ & $2,68 \pm 1,33^{b}$ & $\begin{array}{c}2,94 \pm \\
1,13^{\mathrm{b}}\end{array}$ & $\begin{array}{c}2,90 \pm \\
1,30^{\mathrm{b}}\end{array}$ \\
\hline & SP & $4,39 \pm 0,68$ & $1,26 \pm 0,06^{\mathrm{a}}$ & $\begin{array}{c}1,33 \pm \\
0,44^{\mathrm{a}}\end{array}$ & $\begin{array}{c}0,96 \pm \\
0,78^{\mathrm{a}}\end{array}$ \\
\hline & $\mathrm{CM}$ & $4,63 \pm 0,52^{1}$ & $1,97 \pm 1,14^{2}$ & $\begin{array}{c}2,13 \pm \\
1,16^{2}\end{array}$ & $\begin{array}{c}1,93 \pm \\
1,43^{2}\end{array}$ \\
\hline \multirow[t]{3}{*}{ Chlorophylle $a$} & SS & $2,39 \pm 0,31$ & $5,64 \pm 1,28^{a}$ & $\begin{array}{c}4,66 \pm \\
1,82^{\mathrm{a}}\end{array}$ & $\begin{array}{c}5,66 \pm \\
1,35^{\mathrm{a}}\end{array}$ \\
\hline & SP & $2,95 \pm 0,74$ & $7,60 \pm 0,26^{b}$ & $\begin{array}{l}7,28 \pm \\
0,19^{\mathrm{b}}\end{array}$ & $\begin{array}{c}8,83 \pm \\
1,51^{\mathrm{b}}\end{array}$ \\
\hline & $\mathrm{CM}$ & $2,67 \pm 0,59^{2}$ & $6,62 \pm 135^{1}$ & $\begin{array}{c}5,97 \pm \\
1,84^{1}\end{array}$ & $\begin{array}{l}7,24 \pm \\
2,16^{1}\end{array}$ \\
\hline
\end{tabular}

$\mathrm{SS}=$ Saison sèche $; \mathrm{SP}=$ Saison des pluies, $\mathrm{CM}=$ Concentrations moyennes.

Les valeurs de la même colonne portant des lettres $a$, $b$, en exposant présentent une différence entre les saisons $(p<0,05)$ pour une structure donnée.

Les valeurs de la même ligne portant des lettres 1, 2 en exposant présentent une différence entre les structures $(p<0,05)$.

\section{II-1-3-Relation entre les variables mesurées}

L'examen de la matrice de corrélation entre variables (Tableau III) révèle des relations entre les paramètres physico-chimiques, les sels nutritifs et la chlorophylle $a$.

Le nitrate est moyennement corrélé négativement avec l'oxygène dissous $(\mathrm{r}=-0,80)$ et la transparence $(\mathrm{r}=-0,87)$. Il est moyennement corrélé positivement avec les matières en suspension $(\mathrm{r}=0,78)$, faiblement corrélé négativement avec le $\mathrm{pH}(\mathrm{r}=-0,55)$ et faiblement corrélé positivement avec la température $(\mathrm{r}=0,59)$. Par contre, l'orthophosphate est moyennement corrélé négativement avec les matières en suspension $(r=-0,82)$, mais moyennement corrélé positivement avec la transparence $(\mathrm{r}=0,71)$ et faiblement corrélé positivement avec l'oxygène dissous $(\mathrm{r}=0,59)$. Quant à la chlorophylle $a$, elle est fortement corrélé négativement avec l'orthophosphate $(\mathrm{r}=-0,97)$ (Figure 4) mais moyennement corrélé négativement avec la transparence $(r=-0,72)$. En revanche, elle est moyennement corrélé positivement avec les matières en suspension $(\mathrm{r}=0,85)$ et le nitrate $(\mathrm{r}=0,86)$ et moyennement corrélé négativement avec l'oxygène dissous $(\mathrm{r}=-0,57)$ et l'ammonium $(\mathrm{r}=-0,52)$. 
Tableau 3 : Corrélation entre les paramètres physico-chimiques, les sels nutritifs et la chlorophylle a des eaux des étangs et du barrage la ferme piscicole ATTAH de février à juillet 2017.

\begin{tabular}{|c|c|c|c|c|c|c|c|c|c|c|}
\hline & $\mathrm{T}^{\circ} \mathrm{C}$ & $\mathrm{O}_{2}$ & $\mathrm{pH}$ & Transp & MES & $\mathrm{NO}_{3}{ }^{-}$ & $\mathrm{NO}_{2}^{-}$ & $\mathrm{NH}_{4}{ }^{+}$ & $\mathrm{PO}_{4}{ }^{3-}$ & $\begin{array}{c}\text { Chlo } \\
\text { a }\end{array}$ \\
\hline $\mathrm{T}^{\circ} \mathrm{C}$ & 1 & & & & & & & & & \\
\hline $\mathrm{O}_{2}$ & $\begin{array}{c}- \\
0,88^{*}\end{array}$ & 1 & & & & & & & & \\
\hline $\mathrm{pH}$ & ${ }^{-}, 62 *$ & $0,73^{*}$ & 1 & & & & & & & \\
\hline Transp & $0,85^{*}$ & $0,92 *$ & $0,57 *$ & 1 & & & & & & \\
\hline MES & 0,47 & $0,65^{*}$ & $-0,37$ & $-0,75^{*}$ & 1 & & & & & \\
\hline $\mathrm{NO}_{3}{ }^{-}$ & $0,59 *$ & $-\overline{-} 80^{*}$ & $0,55^{*}$ & $-0,87 *$ & $0,78^{*}$ & 1 & & & & \\
\hline $\mathrm{NO}_{2}{ }^{-}$ & 0,45 & $-0,34$ & $-0,40$ & $-0,22$ & $-0,15$ & $-0,02$ & 1 & & & \\
\hline $\mathrm{NH}_{4}^{+}$ & $-0,21$ & 0,20 & 0,00 & 0,43 & $-0,43$ & $-0,44$ & 0,43 & 1 & & \\
\hline $\mathrm{PO}_{4}^{3-}$ & $-0,37$ & $0,59 *$ & 0,37 & $0,71^{*}$ & $\begin{array}{c}- \\
0,82 *\end{array}$ & $\begin{array}{c}- \\
0,89 *\end{array}$ & 0,22 & $0,53^{*}$ & 1 & \\
\hline Chlo a & 0,38 & $0,57 *$ & $-0,33$ & $-0,72 *$ & $0,85^{*}$ & $0,86^{*}$ & $\begin{array}{c}- \\
0,16\end{array}$ & $0,52 *$ & $\stackrel{-}{0,97 *}$ & 1 \\
\hline
\end{tabular}

Les valeurs en $*$ présentent une corrélation significative à $p<0,05$.

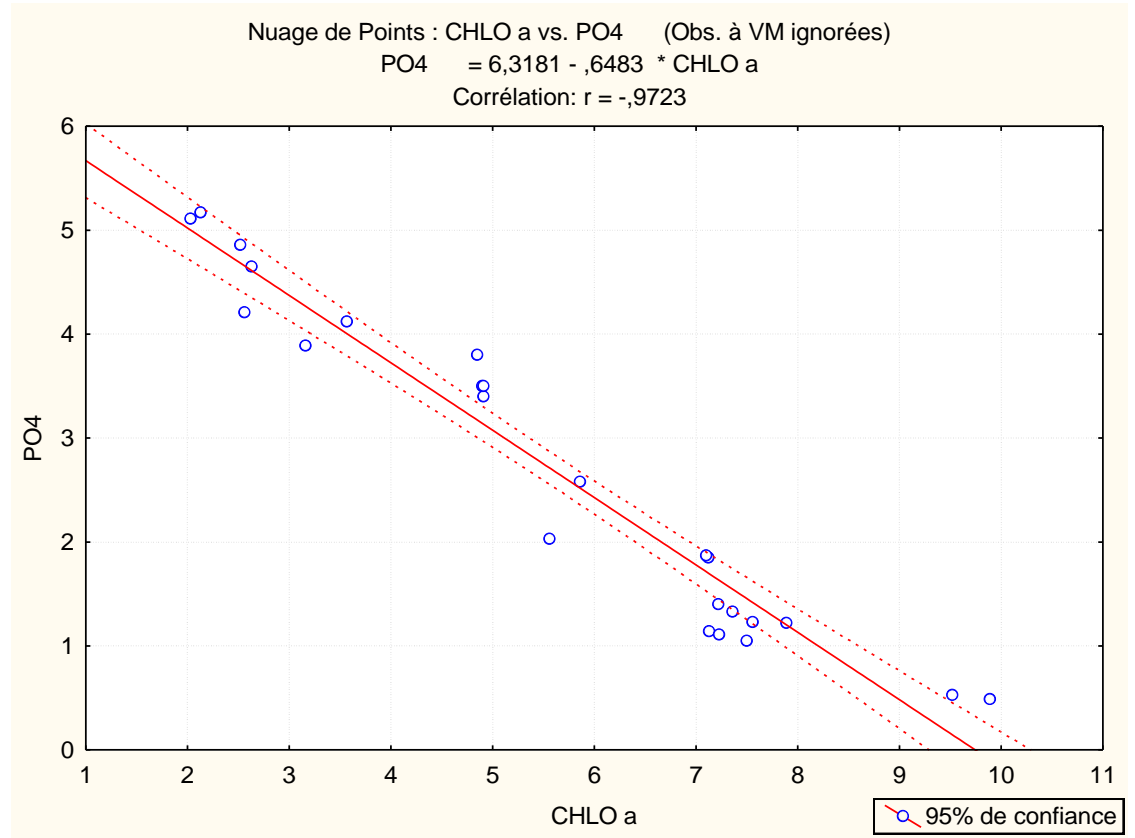

Figure 4 : Corrélation fortement négative entre l'orthophosphate et la chlorophylle a 


\section{II-2-Discussion}

Les résultats de cette étude ont montré que les eaux des étangs de grossissement de $O$. niloticus de la ferme piscicole ATTA sont chaudes, acides, peu oxygénées et faiblement transparentes avec des taux élevés de matière en suspension. Les résultats ont montré qu'il existe une différence saisonnière au niveau de la transparence et des matières en suspension. Les caractéristiques des eaux de la ferme et les variations saisonnières peuvent être attribuées à la température élevée de l'air, aux apports riches en sestons des eaux de ruissellement et à l'activité agricole importante autour de la ferme (Kouassi et al., 2005 ; Atta et al., 2014). Cette étude a également montré qu'il y a une différence significative des paramètres physico-chimiques mesurés dans les étangs et le barrage d'alimentation. Cette différence est probablement due à la profondeur des structures d'une part, et d'autre part, au débit de renouvellement des eaux. En effet, la profondeur des étangs est faibles (1,50 $\mathrm{m}$, dans la partie la plus profonde) par rapport à celle du barrage $(2,20 \mathrm{~m})$. Par ailleurs les eaux du barrage sont continuellement renouvelées par la rivière d'alimentation et la nappe phréatique. Le renouvellement des eaux des étangs est contrôlé pour une gestion rationnelle des eaux. Toutefois, nos valeurs de la température $\left(30,88-31,03{ }^{\circ} \mathrm{C}\right)$, de l'oxygène dissous $(3,73-3,83 \mathrm{mg} / \mathrm{L})$ et du $\mathrm{pH}(6,58-6,89)$ dans les étangs sont comprises dans la gamme tolérable pour O. niloticus dont les valeurs extrêmes tolérables sont de $7-41^{\circ} \mathrm{C}$ pour la température, de $0,1 \mathrm{mg} / \mathrm{L}$ pour l'oxygène dissous, de 5-11 pour le $\mathrm{pH}$, (Plisnier et al., 1988 ; Rakotovao, 2004 ; Hamouda, 2005). En revanche, les valeurs des M.E.S (95,76 - 244,12 mg/L) sont supérieures aux valeurs de MES extrêmes tolérables par $O$. niloticus qui est de $200 \mathrm{mg} / \mathrm{L}$. Aussi, la transparence $(0,00-$ $0,01 \mathrm{~cm})$ dans les étangs est inférieure à la transparence idéale $(20 \mathrm{~cm})$ pour l'élevage du tilapia O. niloticus (Pouomogne, 1998 ; Mfossa, 2007). Ces taux élevés de MES peuvent provoquer des dommages aux poissons en se colmatant sur les branchies et les empêcher de respirer ou de filtrer l'eau pour l'osmorégulation.

L'analyse des taux des sels nutritifs et de la chlorophylle $a$ a montré une différence significative $(p<0,05)$ entre les saisons pour le nitrate, l'orthophosphate et la chlorophylle $a$. Les variations saisonnières observées au cours de cette étude sont probablement dues à l'apport des eaux de ruissellement et des effluents agricoles pendant la saison des pluies (Yao et $a l ., 2009)$. L'étude révèle des taux presque inexistants de nitrite $(0,00-0,01$ $\mathrm{mg} / \mathrm{L})$ dans toutes les structures échantillonnées. Les valeurs obtenues sont inférieures à celles rapportées par Avit et al. (2012) en étangs d'élevage de O. niloticus $(5,33 \pm 2,99 \mu \mathrm{g} / \mathrm{L})$. Les valeurs moyennes de nitrate varient entre $9,31 \pm 1,33 \mathrm{mg} / \mathrm{L}$ et $9,58 \pm 1,16 \mathrm{mg} / \mathrm{L}$. Ces valeurs sont supérieures à celles enregistrées par Ipungu et al. (2015) dans les étangs fertilisés au Congo (0,07 $\mathrm{mg} / \mathrm{L})$. Les faibles valeurs du nitrite et les fortes valeurs de nitrate observées 
peuvent s'expliquer par une oxydation des nitrites en nitrates. Les concentrations d'ammonium $(1,48-1,97 \mathrm{mg} / \mathrm{L})$ et d'orthophosphate $(1,93-$ 2,13 mg/L) déterminées dans la présente étude sont supérieures à celles rapportées par Ntumba et al. (2016) dans les étangs piscicoles des vallées de Kimwenza à Kinshasa $(0,2 \pm 0,09 \mathrm{mg} / \mathrm{L}$ et $0,31 \pm 0,19 \mathrm{mg} / \mathrm{L}$ respectivement pour l'ammonium et l'orthophosphate).. Les variations d'ammomium et d'orthophosphate résulteraient d'une part de la décomposition des déchets et excréments issus des poissons et d'autre part du reste d'aliments industriels non consommés par les poissons (Bahri, 2009). Toutefois, les valeurs résiduelles dans les écosystèmes aquatiques indépendamment des apports exogènes d'aliments sont plus élevées comme le montrent les valeurs mesurées dans le barrage $\left(2,96 \mathrm{mg} / \mathrm{L}\right.$ pour $\mathrm{NH}_{4}{ }^{+}$et $4,63 \mathrm{mg} / \mathrm{L}$ pour $\left.\mathrm{PO}_{4}{ }^{3-}\right)$ où aucun aliment industriel n'a été apporté. Par ailleurs, les concentrations élevées dans le barrage pourraient être la conséquence de l'utilisation des fertilisants dans les activités agricoles menées autour de la ferme.

Les teneurs en $\mathrm{NO}_{2}{ }^{-}$et en $\mathrm{NH}_{4}{ }^{+}$restent inférieures aux seuils pour l'élevage du tilapia, décrits par Ballarin et Haller (1982) qui sont de 2,1 mg/L pour les nitrites et de $2,3 \mathrm{mg} / \mathrm{L}$ pour l'ammoniaque. Les valeurs en $\mathrm{NO}_{3}{ }^{-}$et en $\mathrm{PO}_{4}{ }^{3-}$ sont supérieures aux seuils qui sont de $2,1 \mathrm{mg} / \mathrm{L}$ pour les nitrates et de $0,5 \mathrm{mg} / \mathrm{L}$ pour l'orthophosphate. Selon FAO (2011) une prolifération d'algues sera constatée au-delà de ces valeurs.

Les valeurs de la chlorophylle $a$ obtenues dans cette étude $(5,97 \pm 1,84$ -

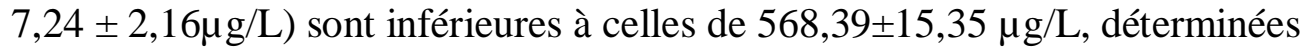
par Imorou Toko et al. (2010) dans des étangs de tilapia en système semiintensif au Bénin et à celles $(40,56 \pm 8,80$ à 94,68 $12,91 \mu \mathrm{g} / \mathrm{L})$ rapportées par Agadjihouèdé et al. (2016) dans le périmètre rizicole de Malanville au NordEst Bénin. Les concentrations en chlorophylle $a$ des étangs $(5,97 \pm 1,84-$ $7,24 \pm 2,16 \mu \mathrm{g} / \mathrm{L})$ sont supérieures à celles du barrage $(2,67 \pm 0,59 \mu \mathrm{g} / \mathrm{L})$. L'apport exogène d'aliments industriels dans les étangs pourrait augmenter la teneur en nutriments de ces étangs entrainant une prolifération d'algues, ce qui justifierait les valeurs élevées de la chlorophylle $a$ observées par rapport au barrage (Mélard, 2006).

La matrice de corrélation établie entre les paramètres physicochimiques, les sels nutritifs et la chlorophylle $a$ a montré une forte corrélation négative $(r=-0,97)$ entre la chlorophylle $a$ et l'orthophosphate. Cette forte corrélation pourrait s'expliquer par le fait que plus l'eau est riche en phytoplancton plus elle s'appauvrit en orthophosphate (Benabdellouahad, 2006). Les corrélations du nitrate, négatives avec l'oxygène dissous et la transparence, et positives avec les matières en suspension, confirment une distribution homogène des MES et des nitrates. 
Les corrélations de l'orthophosphate positives avec l'oxygène dissous et la transparence, et négatives avec les matières en suspension montre une distribution inverse des MES et de l'orthophosphate.

\section{Conclusion}

Dans cette étude, l'analyse des paramètres physico-chimiques a révélé que les eaux des étangs de grossissement de $O$. niloticus de la ferme piscicole ATTA sont chaudes, faiblement oxygénées, chargées en matière en suspension avec une transparence faible et un caractère acide. De tous les paramètres étudiés, seules les valeurs des M.E.S sont supérieures aux valeurs extrêmes tolérables par $O$. niloticus. Ces taux élevés de MES peuvent provoquer un stress chez les poissons élevés dans ces étangs. L'étude des sels nutritifs et de la chlorophylle «a » a montré une différence significative entre les saisons pour le nitrate, l'orthophosphate et la chlorophylle $a$. Les taux en nitrate et en orthophosphate sont supérieurs aux seuils préconisés par la FAO pour les étangs d'élevage. Aussi, les concentrations en chlorophylle $a$ des étangs sont supérieures à celles du barrage. Ceci traduit le faite que l'enrichissement des étangs en nutriments est dû non seulement aux apports exogènes d'aliments mais aussi aux rejets des activités agricoles. Cette étude a montré une corrélation fortement négative entre l'orthophosphate et la chlorophylle «a ». Les paramètres mesurés montrent qu'il y a un début d'eutrophisation pouvant entrainer le stress et la mortalité des poissons à longs terme.

\section{Remerciement}

Les auteurs voudraient exprimer leur sincère remerciement à l'Académie des Sciences, des Arts, des Cultures d'Afrique et des Diasporas Africaines (ASCAD) pour le financement de cette étude.

\section{References:}

1. Abboud J., Aoun J., Jammal B., El Rammouz R. \& Saab A.M. (2015). Effect of chemical accumulation and temperature on fish production in Lebanese water ponds. International Journal of Fisheries and Aquatic Studies, 3(1): 43-47.

2. Agadjihouèdé H., Ahouansou M.S., Montchowui E., Bonou A.C. \& Lalèyè P. (2016). Composition et abondance du peuplement zooplanctonique des étangs piscicoles du périmètre rizicole de Malanville au Nord-Est Bénin. Bulletin de la Recherche Agronomique du Bénin (BRAB) Numéro spécial Agronomie, Société, Environnement \& Sécurité Alimentaire, 1025-2355.

3. Aminot A. \& Chaussepied M. (1983). Manuel des analyses chimiques en milieu marin. CNEXO, Editions Jouve, Paris, 395 p. 
4. Atta K.L., Gogbe T. \& Moussoh A.A. (2014). La problématique de l'approvisionnement vivrier d'une ville secondaire dans le cadre des relations ville-campagne en pays Adioukrou: Dabou. European Scientific Journal, 17 : 293-307.

5. Avit J.L.F., Bony K.Y., Kouassi N.G.C., Konan K.F., Assemian O. \& Allouko J.R. (2012). Conditions écologiques de production de fingerlings de Oreochromisniloticus (Linné, 1758) en association avec le riz WITA 12 en étang, Journal of Applied Biosciences, 59: 42714285.

6. Ballarin J.D. \& Haller R.D. (1982). The intensive culture of Tilapia in tanks, raceways and cages. In: Muir \& Roberts. Recent Advances in Aquaculture, (1): London, 155p.

7. Bahri A. (2009). Contribution à la valorisation des eaux aquacoles dans l'amélioration de la production de l'oignon et de la laitue (cas de Kef es Soltane Ouargla). Mémoire de fin d'études en vue de l'obtention du Diplôme d'Ingénieur d'Etat en Sciences Agronomiques. Université Kasdi-Merbah- Ouargla, 99p.

8. Benabdellouahad S. (2006). Structure, dynamique et typologies physico-chimiques et phytoplanctoniques de l'estuaire du bouregreg (Côte Atlantique Marocaine) Thèse de Doctorat. Université Moammed V, Rabat, Maroc, 293 p.

9. Butler R., Reedyk S., Murrell S. \& Mah B. (2005). Use of barley straw for algal control in prairie dugouts. Agriculture and Agri-Food Canada, ISBN 0-662-42192-2, $43 \mathrm{p}$.

10. FAO. (2011). Méthodes simples pour l'aquaculture. En ligne sur www.fao.org/Passion bassin, consulté le 30-11-2017.

11. FAO. (2014). Fisheries and Aquaculture Information and Statistics Service.Rome

(Italie):FAO.http://www.fao.org/figis/servlet/SQServlet?ds=Aquacult ure \&k1=COUNTRY\&k1v=1\&k1s=107\&outtype=html, consulté le 19-11-2017.

12. FAO. (2017). Cultured aquatic species fact sheets Oreochromisniloticus (Linnaeus, 1758). http://www.fao.org/fishery/culturedspecies/Oreochromis_niloticus/fr, consulté le 13-11-2017.

13. Hamouda I.A. (2005). Contribution à l'étude de l'inversion sexuelle chez une espèce de poisson d'eau douce: Tilapia (Oreochromisniloticus). Mémoire en vue de l'obtention d'un diplômed'ingenieur d'état en Sciences de la mer https://www.memoireonline.com/07/09/2320/m, consulté le 13-112017. 
14. Imorou I.T., Attakpa E.Y. \& Elegbe H. (2010). Performances biologiques, zootechniques et nutritionnelles de Tilapia guineensis en milieux naturel et d'élevage, International Journal of Biological and Chemical Sciences, 4(5): 1629-1640.

15. Ipungu L., Ngoy K., Banze K., Lumfwa K. \& Kafund M. (2015). L'étude de la croissance de Oreochromisniloticus par la fertilisation des étangs: le cas de la ferme NaviunduLubumbashi. Journal of Applied Biosciences, 91: 8503 -8510.

16. Koroleff F. (1976). Determination of ammonia in natural waters as indophenol blue. ICES, C.M. 1969/C 9 HydrComm, 12 p.

17. Kouassi A.M., Tidou A.S. \& Kamenan A. (2005). Caractéristiques hydrochimiques et microbiologiques des eaux de la lagune Ebrié (Côte d'Ivoire). Agronomie Africaine, 2 : 117-136.

18. Koumi A.R., Kimou B.N., Atsé B.C., Ouattara I.N. \& Kouamé L.P. (2015). Fish Feeds Used in Côte d'Ivoire: Nature, Quality, Use and Productivity. Asian Journal of Agriculture and Food Sciences, 3 (2): 225-236.

19. Lorenzen C.J. (1967). Determination of chlorophyll and pheopigments: spectrophotometric equation. Limnology Oceanography, 12:343-346.

20. Mélard C. (2006). Base biologique de l'aquaculture : note de cours à l'intention des étudiants du DES en Aquaculture, Tihange, ULGFUNDP, Belgique, 302 p.

21. Mfossa M.D. (2007). Caractérisation des étangs d'inondation de la plaine des Mbô et analyse des facteurs influençant leur production piscicole Université de Dschang, FASA-Diplôme d'Ingénieur des Eaux, Forêt et Chasses, https://www.memoireonline.com/03/12/5602/consulté le 13-11-2017.

22. Murphy J. \& Riley J.P. (1962). A modified single solution method for the determination of phosphate in natural water. Analytica Chimica Acta, 27:31-36.

23. Ntumba M.J.M., Mbadu Z.V. \& Kilolo K.V. (2016). Evaluation de la gestion des étangs piscicoles des vallées de Kimwenza dans la commune de Mont Ngafula / Kinshasa, R.D. Congo. Congo Sciences, 4 (2) : 124-134.

24. Plisnier P.D., Micha J.C. \& Frank V. (1988). Biologie et exploitation des poissons du lac Ihema (Bassin de l'Akagera, Rwanda). Presses Universitaires de Namur, Namur, Belgique, $212 \mathrm{p}$.

25. Pouomogne V. (1998). Pisciculture en milieu tropical Africain: Comment produire du poisson à coût modéré. Coopération française, Centre d'excellence pour la production, l'innovation et le développement. Presse universitaire d'Afrique, 236p. 
26. Rakotovao R.J.N. (2004). Valorisation des tilapias en charcuterie : fabrication semi-industrielle de saucisses à base de tilapias. Mémoire de fin d'études en vue de l'obtention du diplôme d'ingénieur agronome option Industries Agricoles et Alimentaires. Université d'Antananarivo, Madagascar, 161p.

27. Yao K.M., Métongo B.S., Trokourey A. \& Bokra Y. (2009). La pollution des eaux de la zone urbaine d'une lagune tropicale par les matières oxydables (lagune Ebrié, Côte d'Ivoire). International Journal of Biological and Chemical Sciences, 3(4): 755-770.

28. Yao A.H., Koumi A.R., Nobah K.S.C., Atsé B.C. \& Kouamelan E.P. (2016). Evaluation de la compétitivité des systèmes piscicoles pratiqués en Côte d'Ivoire: gestion, alimentation et production. International Journal of Biological and Chemical Sciences, 10 (3): 1086-1097. 